\title{
Single segment actuator using shape memory alloy for three- directional bending endoscopic tip
}

\author{
Muhammad Raihan Abdul Kadir a, Dyah Ekashanti Octorina Dewi a,b, ${ }^{\star}$, Mohamed Sultan Mohamed \\ Ali ${ }^{\circ}$, Ahmad 'Athif Mohd Faudzi ${ }^{c}$ \\ a Faculty of Biosciences and Medical Engineering, Universiti Teknologi Malaysia, 81310 UTM Johor Bahru, Johor, Malaysia \\ b IJN-UTM Cardiovascular Engineering Center, Institute of Human Centered Engineering, Universiti Teknologi Malaysia, 81310 UTM Johor Bahru, \\ Johor, Malaysia \\ c Faculty of Electrical Engineering, Universiti Teknologi Malaysia, 81310 UTM Johor Bahru, Johor, Malaysia
}

* Corresponding author: dyah@utm.my

\section{Article history}

Received 15 October 2017

Accepted 6 December 2017

\begin{abstract}
Shape Memory Alloy (SMA) is a specially fabricated alloy which is capable of altering its shape based on external stimuli. With this mechanism, SMA can be used as actuator with highly controlled maneuverability and flexibility. This study was aimed to design, fabricate, test and analyze an SMA actuator with three-directional bending capabilities. The design consisted of a set of SMA actuators with a pseudo-elastic alloy bias wire. A silicone rubber sheathe was fabricated to act as a heat buffer. The actuator was activated with a DC current of $0-900 \mathrm{~mA}$ at $70 \%$ PWM duty cycle. Bending and heat tests resulted in positive bending of about $30^{\circ}$ consistently when the silicone rubber sheathe was not applied as cover in the instrument. Temperature readings on the thermal camera are $40^{\circ} \mathrm{C}$. With the silicone rubber sheathe attached, the bending angle is halved over the entire instrument to $12^{\circ}$, but with a smaller decrease in recorded temperature of the main bodies. This SMA-based actuator is prospectively used to improve the maneuverability and flexibility of an endoscopic tip.
\end{abstract}

Keywords: Endoscopic tip, single segment, bending, actuator, shape memory alloy

\section{INTRODUCTION}

Shape Memory Alloys (SMA) has been widely used in many industries, especially in the fields of aeronautics, automotive, and medicine. SMAs are a specially fabricated alloy which is capable of altering its shape based on external stimuli. These stimuli can either be in the form of electrical current or even heat. The crystal lattice structures of SMAs alternates between austenitic (stiff phase or memorized shape) and martensitic phase (soft phase) based on the aforementioned stimuli, such as heat and electrical current. This property gives SMAs an additional advantage of having a high force to weight ratio. For this reason, actuators from SMAs are applicable for high force purposes, such as specialized structures for automotive and aeronautics applications. However, once the bending has taken place in order to return to its original shape, a bias spring is needed to push it back by acting as an opposing force once the SMA is in the martensitic phase (Jani et al., 2014).

Nitinol is most commonly used SMA that has been used as actuator for solenoids and servo motors. With temperature-dependent shape deformity characteristics, Nitinol has its unique shape memory effect and super-elasticity features. For generating an effective Nitinol-based actuator, a Pulse Width Modulation (PWM) circuit is used as electrical heating of Nitinol to distribute the heat evenly along the actuator. A PWM operates by turning the power source on and off at specific frequencies known as duty cycles. Duty cycles is the percentage of time that the circuit is turned on in one cycle. If the PWM circuit has $100 \%$ duty cycle, then the circuit is entirely on. But, if the circuit has a $0 \%$ duty cycle, then the circuit is not allowing any current to flow through it. Whereas, a $50 \%$ duty cycle means that the circuit is on for one half of the cycle, but off for the other half. PWM circuits are usually used in precise control of instruments from the speed of computer fans to the power supplied to a DC motor of linear actuators. In the case of SMAs, if the actuator is supplied with current that has not gone through modulation, excessive and uneven heating is supplied to the SMA, causing permanent damage. The PWM also functions to better control the movement of the actuator by varying the duty cycle of the supplied current. Thus, the higher the duty cycle, the stronger the output power (Ma et al., 2003).

In various biomedical technologies, SMAs have been preferred due to its corrosion resistance, biocompatibility and inertness characteristics (Petrini et al., 2011). With these properties, SMAs can be potentially developed as biomedical materials for implants or surgical and interventional devices, especially in orthopedic, dentistry, and cardiovascular applications (Chan et al., 2016). The applications may range from stent implants to treat blocked coronary arteries, orthodontic brackets for crooked dental treatment, active needle, guide wires and active catheters in coronary artery diseases, to a number of endoscopic systems to reach the internal body area with minimal incision for observation, intervention, biopsy, and mini-surgeries with active high degree of bending flexibility and accurate maneuverability (Park et al., 2011). Specifically, since Nitinol is made of Nickel and Titanium materials, this alloy has non-ferromagnetic characteristics that enable for interaction with magnetic-based devices (Zainal et al., 2015). Therefore, Nitinol can be prospectively safe for many biomedical applications, especially in conjunction with Magnetic Resonance Imaging (MRI) procedures. However, its effects on image artifact and noise generation are still under observation (Melzer et al., 2009). 
One of significant SMA applications in biomedical technology is instrument for surgical robotics (Taylor et al., 2016). The function is basically for bending and actuating the tip to navigate through the complex structures of the human body. The main task of SMAs in these types of applications is to improve the dexterity and maneuverability of the surgeon. The obvious applications are endoscopes for diagnosis of gastrointestinal diseases and treatment of heart diseases.

Along with the advancements miniaturization technology, endoscopic system has grown into instrument for Minimally Invasive Surgery (MIS) technology (Bodner et al., 2005). Endoscope has been used to reach the internal body area for tissue observation and manipulation by cutting, drilling, or grasping the tissues, either for surgery, biopsy, treat, or cauterize any infected wounds. For internal visualization aids, imaging system is commonly used to navigate the direction and maneuver of the endoscope tip. Endoscope is also usually built as long tubular instrument to fit in small openings or perforations. With the advent of mini-surgical manipulator technologies, endoscope has also been used in the MIS to minimize tissue damage and improve healing rate of the patient. However, the drawbacks are that rigidity in the endoscopic tip making it lacking of force feedback and limiting the flexibility and capabilities of the surgeon in accessing the target. To solve these issues, some endoscopes have been equipped with actuators to bend to reach the areas of interest or allow the surgeon to perform surgical procedures more easily (Haga et al., 2005).

For procedures like gastroscopies, endoscopic tip actuation is considered to be adequate. However, for other specialized procedures, such as laminectomies, cardiac surgery, and neurosurgery, more flexibility and maneuverability are needed. Due to these limitations, the work of (De Sars et al., 2010) introduced the use of SMA actuation in the design and control of active endoscopic system in a snake-like multi-degree of freedom structure actuated by thin Nitinol springs mounted in an antagonist configuration and directly integrated in the structure of the endoscope. Also, the choice of control scheme has been emphasized to play important role in providing trade-offs between the actuator dynamic characteristics, energy consumption, and structure durability. Other studies in development of endoscopic instruments have also shown their bending capabilities across the entire endoscopic instrument.

In the work of (Ho et al., 2011) in Neurosurgical robots, Nitinol strips was used to actuate each segment of the flexible endoscopic instrument. The instrument consisted of a brass body connected by mechanical fixtures. Each segment had $\pm 30^{\circ}$ bending angle using antagonistic Nitinol strips. A Power Width Modulator PWM switching circuit controlled the Nitinol actuator. However, miniaturization became a problem, since the main body of the actuator was fabricated from brass. As the brass body required mechanical fixture to bend, fabrication became a big issue. This was because the brass parts are hard to fabricate. Specialized equipment, such as computer controlled drills, were needed given that the size of the instrument was only 1.2 $\mathrm{cm}$ in diameter. Another drawback of the system was the fact that each individual segment of the instrument was only capable of 1 degree of freedom for each segment. This problem may severely limit the maneuverability of the surgeon, thus lowering the success rate of the surgery, especially for sensitive procedures in the spine and heart.

Another work of (Zaiter et al., 2016) have shown a promising encounter in the use of a new generation of SMA in micro-positioning stage. In this study, the planar spring actuators were used to generate 3 degree of freedom movements and thermo-mechanical training with electrical current induced heating has been capable of moving a small object for microscopic scanning applications.

This study was aimed to design and fabricate a new bending system for endoscopic tips with better angle bending using SMAs as actuators. The system consisted of three SMA actuators connected to a main body to prevent any unwanted activation of the other actuators via heat conduction and a super-elastic backbone to act as a bias spring. This study consisted of conceptual design of the instrument, fabrication of individual parts of the instrument, assembly of the instrument and testing of the instrument through bend testing to obtain the bending angle and observation through thermal camera to attain the thermal characteristics of the instrument.

\section{METHODOLOGY}

\section{Design}

The system is composed of main body, super-elastic backbone, and Nitinol actuators. The design of components is illustrated in Figure 1. The main body (1.a) was comprised of two parts, top and bottom. The main body functioned as separator among actuators to prevent from any heat conduction affecting and activating the adjacent actuators, as the SMA actuators were principally activated by the heat. The main body parts were designed using 3D model conceptual design. Solidworks, a 3D design software for engineering and design applications, was used. A 3D printing software, 3DSprint, was used to set the placement and thickness of the supports. The software transferred the data to the $3 \mathrm{D}$ printer for fabrication process.

The super elastic backbone (1.b) was made of alloy with a diameter of $0.49 \mathrm{~mm}$. Each tip of this super-elastic backbone was connected to the top and bottom parts of the main body. This backbone acted as a bias spring that functioned to push the instrument back to its original shape once the bending ended. This was designed because each SMA actuator was only capable of bending in a single direction.

The Nitinol actuators (1.c) were comprised of 3 parts, actuator A, $\mathrm{B}$, and $\mathrm{C}$. It functioned as the source of actuation for the system. The actuators were made of $\mathrm{M}$ type Nitinol alloy with heat treatment fabrication process in a transformation temperature of $40^{\circ} \mathrm{C}$ and diameter of $0.49 \mathrm{~mm}$. The Nitinol actuator supplied the force for generating the bending. Each actuator was connected to the PWM circuit via copper crimping mechanically attached to the tips of the actuators.

The PWM circuit was developed using an Arduino microprocessor. The Arduino micro-processor played role as a PWM circuit with the duty cycles set by the user in the Arduino compiler. Since Nitinol was used as the actuator, normal solder could not be used to connect the actuator to the circuit due to the presence of Titanium Oxide on the surface of the Nitinol. For this reason, copper crimping was used to form an electrical connection. In this regard, resin was utilized to reinforce the connection, because the mechanical copper crimping could not be able to hold the wires and the SMA actuators. However, before the copper crimping could be used, the surface of the Nitinol actuator needed to be cleaned using sand paper.

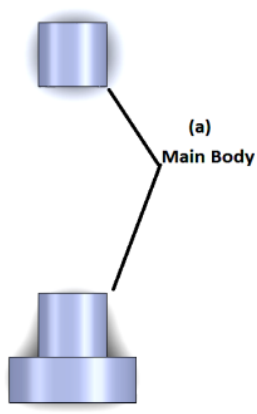

(b) Super-elastic Backbone

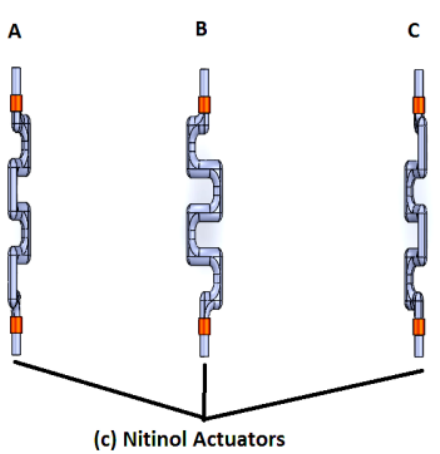

Fig. 1 Individual components of the proposed SMA-based actuation system that consist of (a).Main body, (b).Super-elastic backbone, and (c).Nitinol actuator which consist of actuator A, B and C. 


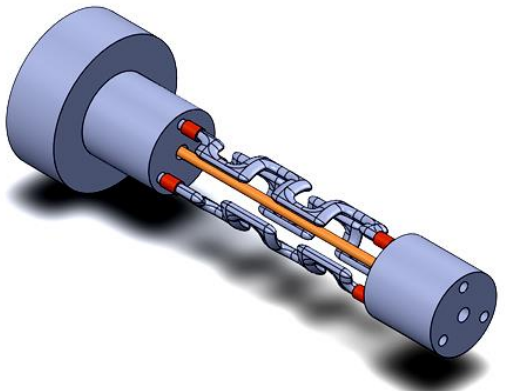

Fig. 2 Assembled SMA-based actuation system.

A silicone rubber sheathe was designed meant to protect the heat to dissipate to its surrounding. As this sheathe was fabricated using Shin Etsu silicone rubber, a moulding system was also designed to accommodate the rubber fabrication process. The moulding system design was made in the same way as the main body in (1.a).

The overall assembled SMA actuation design is given in Figure 2. The actuators were connected to the main bodies via an insertion hole in the main body. The pseudo-elastic backbone was attached to the main body in the same way as the actuators. Each of the individual parts was attached among other by Cyanoacrylic resin to further ensure the connections among adjacent actuators and every individual part.

\section{Training of shape memory alloy}

Super elastic alloys are variants of shape memory alloys. Though they are the same type of alloy as Nitinol which consists of Nickel and Titanium, it is different from SMAs in which the transformation temperature is much lower than room temperature (Paulo et al., 2015). In our case, the transformation temperature of the pseudo-elastic alloy was at $-4^{\circ} \mathrm{C}$. At room temperature, when the pseudo-elastic backbone was deformed or bent in any way, the stress from the deformation transformed the austenitic phase to the martensitic phase causing increased strain at a constant stress.

Once the deformation was done, the martensitic phase reverted back to austenitic phase. Essentially, this had caused the material to retain its original shape once the applied stress was removed, thus it became pseudo-elastic or super-elastic. In this case, the pseudo-elastic alloy was intended to be used as a bias spring due to the fact that the alloy could retain its original straight shape once the Nitinol alloy actuators were no longer activated (Humbeck et al., 1995).
1

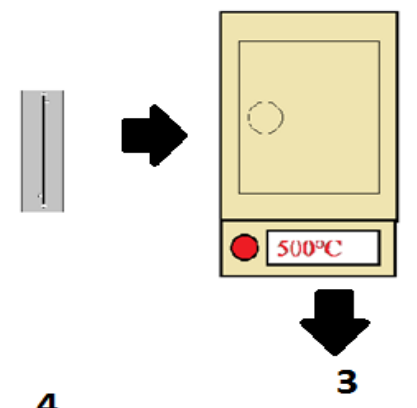

4

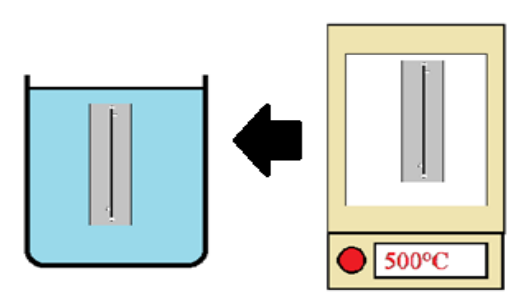

Fig. 3 Actuator shape training process with Step 1 (Setting the Nitinol wire on the aluminium mould), Step 2 (Pre-heating the furnace), Step 3 (Placing the mould with the actuator into the furnace), and Step 4 (Quenching the mould with the actuator).
Nitinol actuators required special heat treatment to "memorize" certain shapes. An aluminum mold was used to set the shape of the Nitinol actuators with steel screws to fasten the Nitinol wire to the mould. When the shape training was done, the Nitinol actuators changed to the shape set in the Aluminum mould when heated to the activation temperature. The steps are given as below:

1. During this process, the actuator was set down into the aluminum mould to ensure that the shape did not deviate from the desired shape during the heating process.

2. A furnace was pre-heated to $500^{\circ} \mathrm{C}$.

3. Once the Nitinol was set down on the mould, it was placed in a furnace which was pre-heated to $500^{\circ} \mathrm{C}$ for 15 minutes.

4. The mould with the actuator attached was quenched in distilled water.

The process was then repeated 3 times to ensure that the shape training was effective enough to be 'memorized'. Figure 3 shows a simplified diagram of the shape training process for the Nitinol actuators.

\section{Fabrication of the main body}

The Main Body was comprised of 2 parts, top and bottom. Once the Main Body design was made, the Main Body was then fabricated using a 3D printer. The printing process applied a Projet 1200 3D printer with suitable printing material of Visijet FTX Green (Microsla, 3D Systems, Inc, North America). The printing setting was using a base size of $5 \mathrm{~cm}$ by $3 \mathrm{~cm}$, a maximum height of $10 \mathrm{~cm}$, and a resolution of $0.02 \mathrm{~mm}$. With such high resolution setting, we could fabricate small insertion holes to fit with the SMA actuators and the pseudo-elastic backbone.

The printing material was solidified using a UV laser in a specific shape to produce the desired shape. Once the first phase of the printing process was done, the Main Body parts were then cleaned using isopropyl alcohol to remove excess uncured Visijet printing liquid on the surface of the Main Body parts. Finally, the Main Body parts were cured using a UV lamp to ensure that the surface was properly cured and hardened. The tips of the Nitinol actuators and super-elastic alloy backbone were then attached to the Main Body parts using Cyanoacrylic resin.

\section{Fabrication of silicone rubber sheathe}

The silicone rubber sheathe was fabricated due to the concerns that the Nitinol actuators heated up to above safe temperatures which was $42^{\circ} \mathrm{C}$ (Yarmolenko et al., 2011). Silicone rubber was chosen due to its ease of fabrication, biocompatibility, and low heat conduction which made it suitable to act as a heat buffer for the instrument. In fabricating the silicon rubber sheathe, a mould was prepared as a negative mould to create sheathe in specified shape and size by using 3D printing technique, in the same way as that of the fabrication for the Main Body. A 3D model was made from Solidwork software and 3DSprint software was used to set the placement and thickness of the supports and transfer the final design to the $3 \mathrm{D}$ printer.

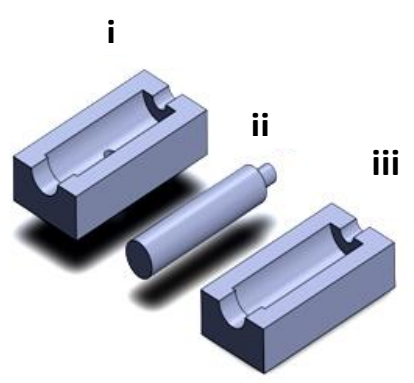

(a)

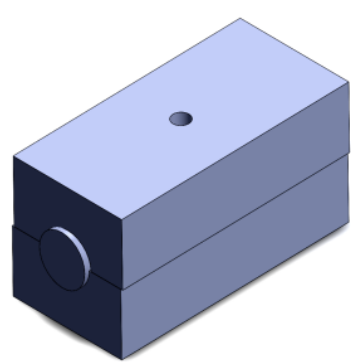

(b)
Fig. 4 Mould design for fabrication of silicone rubber sheathe consisting of 3 parts (a) open-parts i, ii and iii in separated version. (b) close-parts in assembled form. 
The moulding system consisted of 3 parts, part i, ii, and iii, as shown in Figure 4(a). Part $i$ and iii were the outer (upper and lower) parts of the mould which determined the outer diameter of the silicon rubber sheathe, while part ii was the inner part of the mould which determined the inner diameter of the silicon rubber sheathe. With this design, the mould was aimed to produce in $50 \mathrm{~mm}$ long with an outer radius of $7 \mathrm{~mm}$ and an inner diameter of $6 \mathrm{~mm}$ of the silicone rubber sheathe.

The silicone rubber sheathe was made from two parts of Shin Etsu Silicone KE-1603-A/B (Shin-Etsu Chemical Co., Ltd. Tokyo, Japan). The two parts were mixed with 1:1 weight ratio. Before the mixture was poured into the mould, the mould was sprayed with a silicone rubber release agent. This was meant to make the separation between the mould and the cured silicon rubber sheathe easier, as the release agent lubricated the contact surface between the mould and the silicone rubber sheathe. The mixture was poured into a mould and cured to harden for 24 hours. In the beginning of the curing process, the parts were de-gassed with vacuum pump to remove the air bubbles. After cured, the silicon rubber sheathe was then removed from the mould for cover fitting into the instrument, as illustrated in Figure 5.

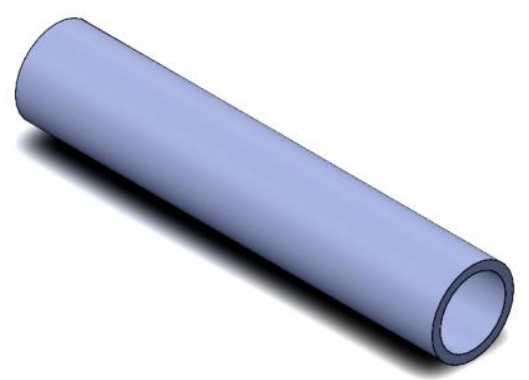

Fig. 5 Schematic design of the silicone rubber sheathe after the cured and removed from the mould system.

\section{Thermal current test}

The instrument was tested in terms of current to determine the most conducive current which produces the highest angle of bending for the instrument. The produced current for the highest bending angle was controlled not to exceed the safe threshold temperature of $42^{\circ} \mathrm{C}$, which is the minimum safe temperature for any medical equipment. The tests were done using a PWM generator at a constant $70 \%$ duty cycle, while varying the current from $0 \mathrm{~A}$ until $1 \mathrm{~A}$. A thermal camera with a maximum of $45^{\circ} \mathrm{C}$ and a minimum detected temperature of $20^{\circ} \mathrm{C}$ were used to observe the temperature increment.

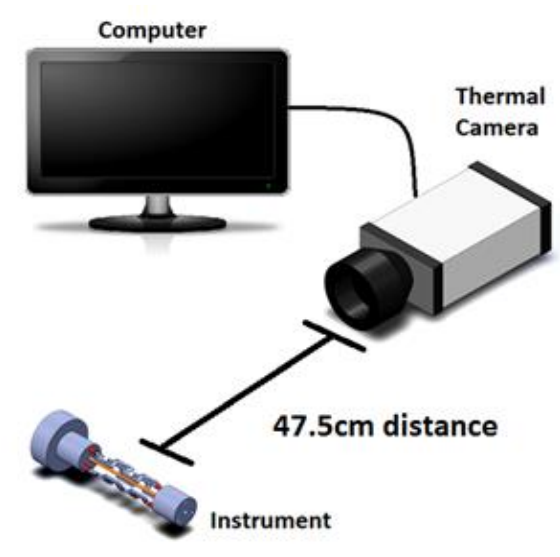

Fig. 6 Schematic experimental setup of thermal current test and angle current test.

The thermal camera was set at a distance of $47.5 \mathrm{~cm}$ to record the entire instrument and measure the bending. The limit was set between $20^{\circ} \mathrm{C}$ to $45^{\circ} \mathrm{C}$. The lowest temperature in the test area was $20^{\circ} \mathrm{C}$ and the transformation temperature of the Nitinol actuator was $40^{\circ} \mathrm{C}$. This setting is preferred due to camera constraints. If the temperature limit was set too low, then the thermal camera only detected the entire instrument as white or high temperature. If the temperature limit was set too high, then the thermal camera only detected the entire instrument as blue or low temperature. In these cases, the thermal characteristics of the instrument would become harder to determine. Figure 6 illustrates the schematic experimental setup for the thermal current test. To test the temperature increasing when the actuator was activated and the effectiveness of the silicone rubber sheathe in reducing the temperature of the instrument during bending, 2 types of experimental settings, covered (with silicone rubber sheathe) and uncovered (without silicone rubber sheathe), were conducted. The heat area distributions in the Main Body A, the Main Body B, and the actuator segment were analyzed.

\section{Angle current test}

The same experimental setup in the thermal current test was also applied for angle current test. The difference is that the thermal camera was replaced by a video camera for bending observation. Figure 6 also shows the schematic experimental setup for the angle current test. The current supply to the instrument was varied from $0 \mathrm{~A}$ to $1 \mathrm{~A}$ while undergoing a PWM signal with $70 \%$ duty cycle. The camera was set at a distance of $47.5 \mathrm{~cm}$ from the instrument to ensure that the entire instrument and the bending were in the footage frame.

The video camera footage was used to analyze the bending angle. The analysis was done by separating the footage to individual frames and the angle of bending was calculated using image processing software by analyzing the footage of the instrument before bending and the footage of the instrument after bending. These tests were done with and without the silicone rubber cover to see the difference in the bending angle of the instrument in each set up. The tests were done for all 3 actuators and the average was calculated from the results.

\section{RESULTS AND DISCUSSION}

\section{Thermal current analysis}

The thermal current results were analyzed by dividing the analysis of the instrument to Main Body A, Main Body B, and the Actuator segment. The heat area distribution between the Main Body A and the Main Body B were calculated using thermal camera software and spatially mapped in the image. From the thermal camera, the software automatically calculated the average temperature per area distribution, while the actuator temperature was calculated by obtaining the average between the recorded points on the actuator. Figure 7 reveals the temperature distribution and analysis in several different areas of the instrument. From this experiment, it explains that the heat was mostly concentrated into the connection between the actuators and the wires. The metallic crimping and resin that were used to bind the actuators and the wires produced more heat than the actuators. This might be due to the copper crimping was not producing a secure electrical connection, or due to the presence of the resin which might act as a resistor and produce unwanted heat. Improvements can be done by replacing the connections with better connector, such as silver epoxy resin.

From Figure 7, we can also indicate that the measured instrument temperature mapping was below $42^{\circ} \mathrm{C}$ for both uncovered (with silicone rubber sheathe, as in Figure 7a) and covered (without silicone rubber sheathe, as given in Figure 7b). This initial in-vitro experiment shows that the instrument temperature range is acceptable for the biomedical safety range and capable of bending without any risk of producing any damage to the surrounding tissues due to heat exposure, even without the silicone rubber sheathe.

In temperature mapping comparison between covered (without silicone rubber sheathe, as in Figure 7a) and uncovered (with silicone rubber sheathe, as in Figure 7b), the silicon rubber sheathe cover has shown to isolate the heat circulation to dissipate outside and distribute more heat from the Main Body A and the Main Body B into the actuator, thus trapping the hot air in the instrument and causing a rise in the actuator temperature. In contrast to the covered actuator version, the temperature mapping in the uncovered actuator has demonstrated 
that the excess heat could be dissipated into the open air, resulting in much lower temperature in the actuator. It seems that the actuator temperature was considerably not much affected by the increasing heat in the Main Body A and the Main Body B. This finding may drive for reconsideration of using silicone rubber sheathe as the actuator cover material that functions as heat protection, or redesigning the silicone rubber sheathe for air circulation system or heat dissipation mechanism in the cover.

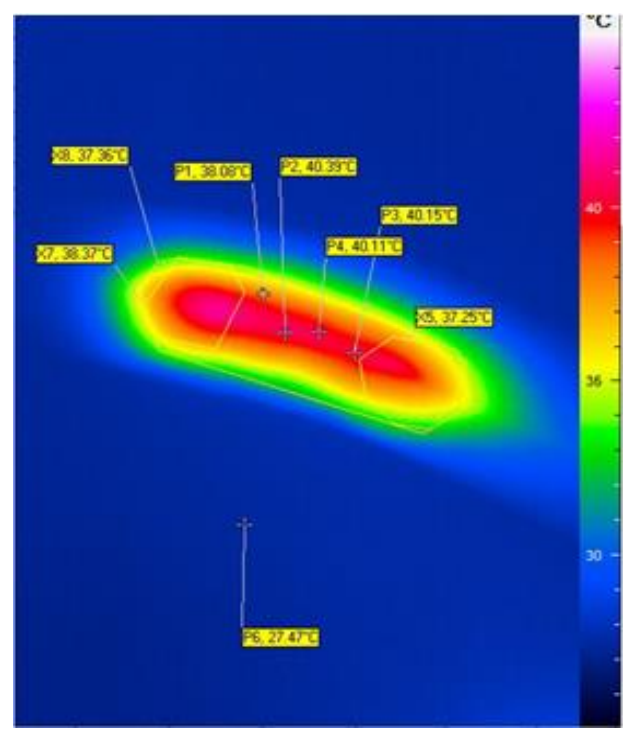

(a)

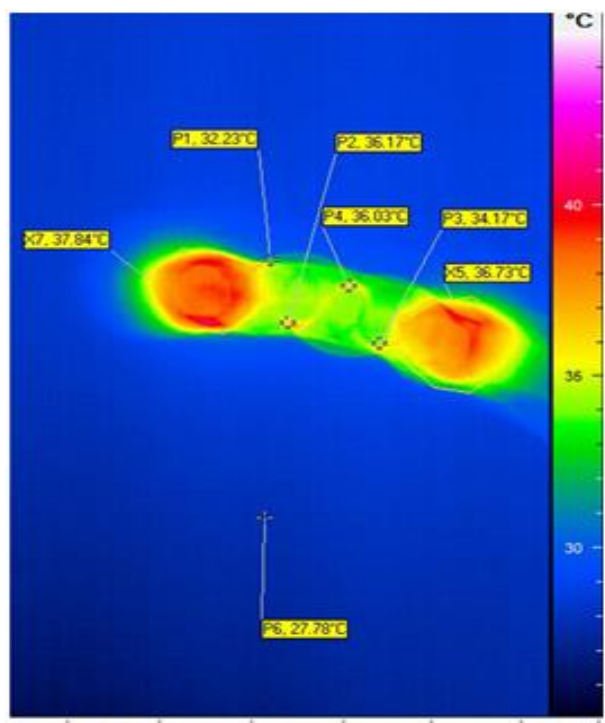

(b)

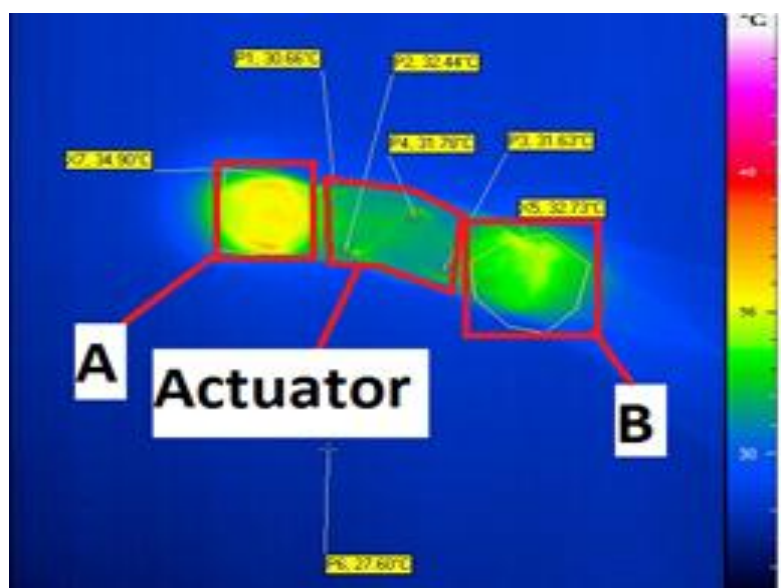

(c)

Fig. 7 Profile of thermal current tests at (a) Covered instrument thermal current test (b) Uncovered instrument thermal current test (c) Analysis of thermal data by dividing the heat area into separated sections.

Besides temperature mapping from the thermal camera, the thermal current test can also associate the current increase relative to the rise of the temperature. Figure 8 to Figure 10 show the plots of the thermal mapping relative to the current for both covered (with silicone rubber sheathe) and uncovered (without silicone rubber sheathe) thermal current tests. From the plots, similar to the abovementioned analysis, we can infer that the addition of the silicone rubber sheathe have raised the temperature of the instrument mostly at the actuator area.

In Figure 8, it can be revealed that both covered (with silicone rubber sheathe) and uncovered (without silicone rubber sheathe) settings have considerably similar thermal characteristics for current range of $0 \mathrm{~mA}$ to $400 \mathrm{~mA}$. Emphasizing the abovementioned analysis on the use of silicon rubber sheathe, the temperature of the covered (with silicone rubber sheathe) actuator increased substantially for the current range of above $400 \mathrm{~mA}$. Actually, the temperature of the uncovered (without silicone rubber sheathe) actuator also increased quite greatly as expected, however, to some extent, the temperature profile was still lower than that of covered version. This finding may suggest for further observation of suitable current range in the actuator activation and their matching with the actuator sheathe design, sheathe material selection, bending performance of the actuator, as well as the application of the actuator.

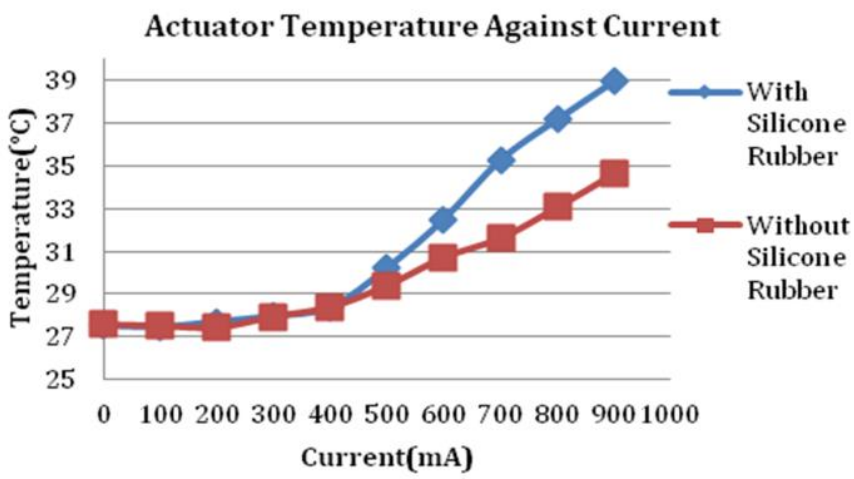

Fig. 8 Thermal test comparison for covered (with silicone rubber sheathe) and uncovered (without silicon rubber sheathe) settings in actuator segment with plot of temperature against current level.

Figure 9 demonstrates the thermal characteristics in the Main Body A relative to the current, comparing the covered (with silicone rubber sheathe) and uncovered (without silicone rubber sheathe) settings. We 
can infer that the temperature in Main Body A was slightly lower for the covered (with silicone rubber sheathe) version for the current range of $0 \mathrm{~mA}$ until $700 \mathrm{~mA}$. However, the temperature characteristics were similar for the test on the current of above $700 \mathrm{~mA}$. Figure 10 presents the thermal characteristics in the Main Body B relative to the current, comparing the covered (with silicone rubber sheathe) and uncovered (without silicone rubber sheathe) settings. In the Main Body B, both settings gave nearly the same results for all current setting.

From these measurements, we can see that the silicone rubber sheathe is successfully able to lower the surface temperature of the instruments at both Main Body A and Main Body B. But, the use of the silicon rubber sheathe has shown to increase the temperature of the actuator segments for current application at $700 \mathrm{~mA}$. Yet, the temperature difference was only $1^{\circ} \mathrm{C}$ to $2^{\circ} \mathrm{C}$, which is not too large compared to the difference in temperature in the actuator region when the instrument was equipped with and without the silicone rubber sheathe.

Main Body A Temperature Against Current

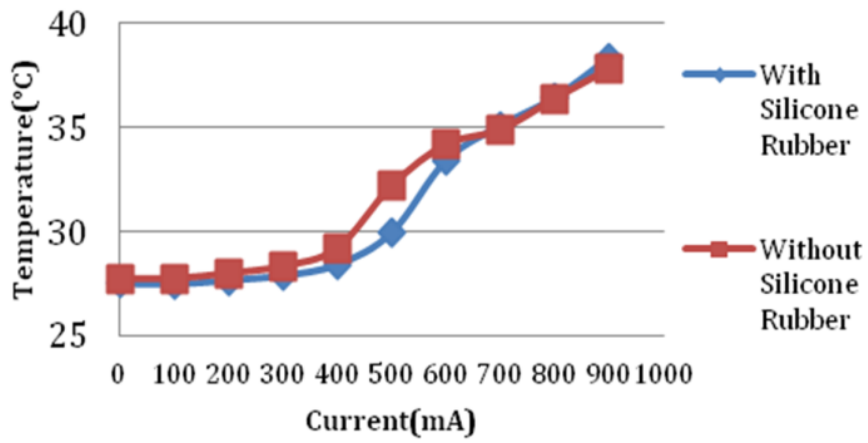

Fig. 9 Thermal test comparison for covered (with silicone rubber sheathe) and uncovered (without silicon rubber sheathe) settings in Main Body A segment with plot of temperature against current level.

Main Body B Temperature Against Current

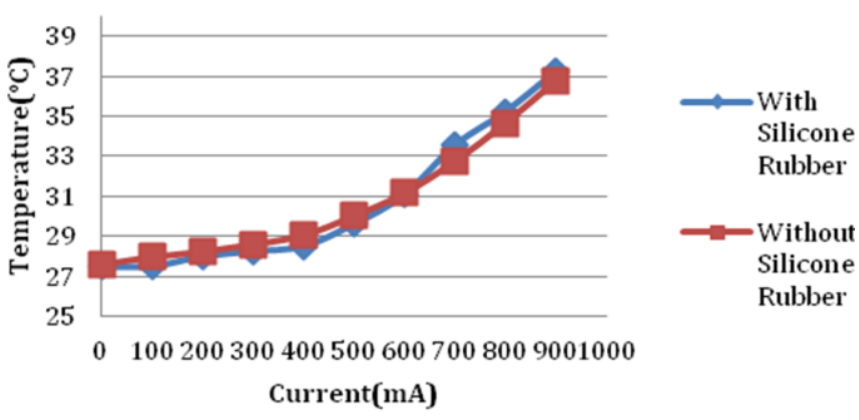

Fig. 10 Thermal test comparison for covered (with silicone rubber sheathe) and uncovered (without silicon rubber sheathe) Main Body B segment with plot of temperature against current level.

\section{Angle current analysis}

A video camera footage was used to analyze the bending angle by capturing the images and calculating bending differences between before (initial state) and after bending (maximum bending). The images were obtained from frame by frame analysis of the video recording during the bending testing. Image processing software was used to analyze the angle of bending of the instruments at different current intervals.

Figure 11 and Figure 12 present the angle current tests in the initial state (a) and maximum bending angle (b) that the actuator could obtain when the current was raised until $700 \mathrm{~mA}$ for both tests in covered and uncovered with silicone rubber sheathe. The first blue line represents the initial angle, while the second blue line represents the angle after the bending. The angle was measured by connecting both blue lines, and creating angle from meeting point of the first to the second blue lines. Figure 13 shows the plot of angle of bending against current level when the actuators were activated.

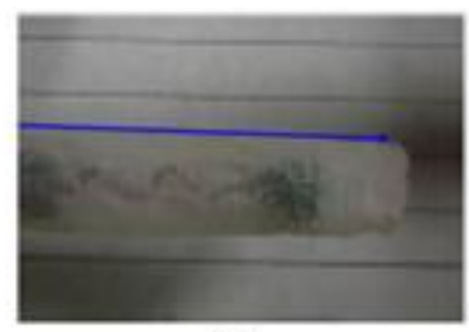

(a)

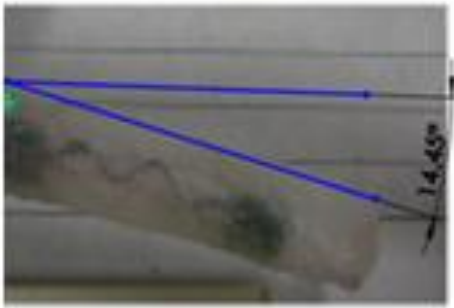

(b)

Fig. 11 Angle current test results for covered (with silicone rubber sheathe) setting (a) Image in initial state (b) Image of bending angle.

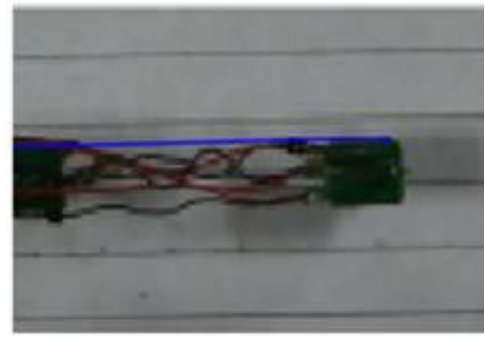

(a)

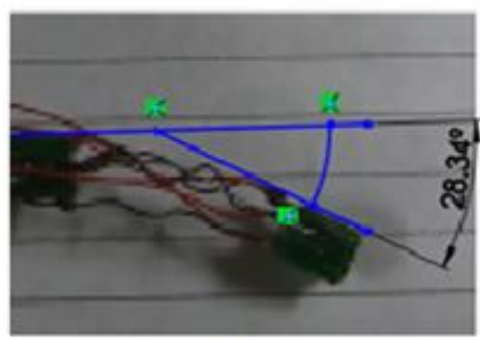

(b)

Fig. 12 Angle current test results for uncovered (without silicone rubber sheathe) setting (a) Image in initial state (b) Image of bending angle.

By comparing the angle current relative to the bending results between the covered setting in Figure $11 \mathrm{~b}$ and uncovered setting in Figure $12 \mathrm{~b}$, it signifies that the maximum bending angle in covered setting provided lower bending angle of only $12^{\circ}$ compared to that of maximum bending angle in the uncovered setting without the silicone rubber sheathe with a bending angle of $30^{\circ}$. This occurs mostly due to the elasticity characteristics of the silicone rubber sheathe has resisted the actuator bending capability. Although the bending capability decreases, the bending profile of the covered setting still reflects similar to that of the uncovered setting, but in smaller angle values, as shown in Figure 13. This result also shows the possibility of controllability feature. However, further study is still needed. Additionally, Figure 13 also demonstrates that the current of $700 \mathrm{~mA}$ at $70 \%$ duty cycle is the optimum level of current to produce maximum amount of bending. The experiment also revealed that any further increase in the current above $700 \mathrm{~mA}$ did not increase the bending angle. This finding was based on our observation over all three actuators of the system that we used in the study. We can also infer that the current of $700 \mathrm{~mA}$ and $70 \%$ duty cycle is compatible for all three actuators for both settings. 


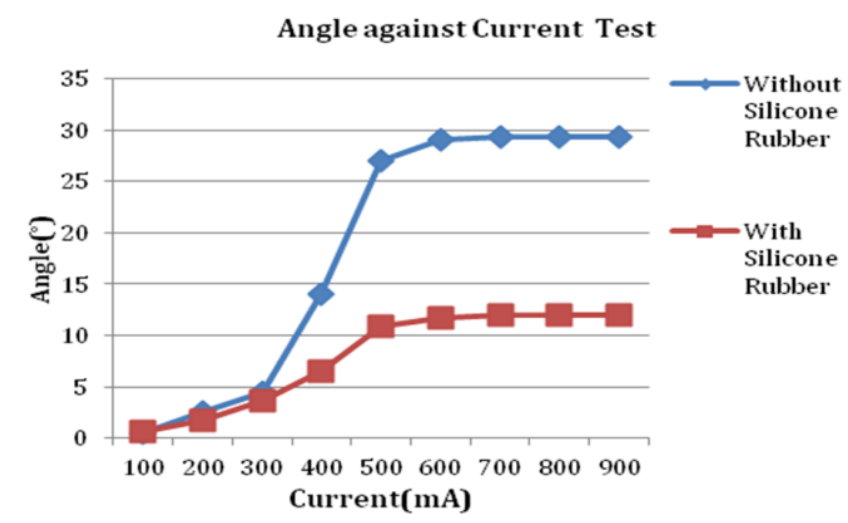

Fig. 13 Plot of angle of bending against current level for both covered and uncovered test.

\section{CONCLUSION}

SMA-based actuator system has been developed and tested. The system that consists of main body, super-elastic backbone, and Nitinol actuators, were fabricated through 3D printing and SMA training. A silicon rubber sheathe was fabricated for surface heat protection. Thermal current and angle current tests were done to evaluate the performance of the system by comparing the covered (with silicone rubber sheathe) and uncovered (without silicone rubber sheathe). The thermal current tests confirm that the measured instrument temperature mapping was still within the safe ranges of temperatures. However, the use of silicone rubber sheathe seems to decrease the main body temperature, but increase the actuator temperature.

The angle current tests clarify that the instrument shows positive bending with the most suitable current of $700 \mathrm{~mA} \mathrm{DC}$ power at $70 \%$ duty cycle. The maximum bending angle was $30^{\circ}$ for the uncovered setting, but only $12^{\circ}$ for the covered setting with the silicone rubber sheathe. This shows that the use of the silicone rubber sheathe has decreased the maximum angle of bending. Therefore, further study on defining the best material and design for the heat protector needs to be done in the future.

\section{ACKNOWLEDGEMENT}

This work was financially supported by the Universiti Teknologi Malaysia and Ministry of Higher Education of Malaysia grants under FRGS Grant R.J130000.7845.4F764 and AUNSEED Net Grant R.J130000.7323.4B187.

\section{REFERENCES}

Jani J. M., Leary M., Subic A., \& Gibson M. A., 2014. A review of shape memory alloy research, applications and opportunities. Mater Des, 56, pp. 1078-1113.

Ma N. \& Song G., 2003. Control of shape memory alloy actuator using pulse width modulation. Smart Mater Struct 12(5), pp. 712-719.

Petrini L. \& Migliavacca F., 2011. Biomedical Applications of Shape Memory Alloys. J. Metall. 2011(2011), Article ID 501483.

Chan B. Q. Y., Low Z. W. K., Heng S. J. W, Chan S. Y., Owh C., \& Loh X. J., 2016. Recent Advances in Shape Memory Soft Materials for Biomedical Applications. ACS Appl. Mater. Interfaces, 8 (16), pp. 10070-10087.

Park A.E., Lee T.H. 2011. Evolution of Minimally Invasive Surgery and Its Impact on Surgical Residency Training. In: Matteotti R., Ashley S. (eds), Minimally Invasive Surgical Oncology (pp. 11-22). Berlin: Springer.

Zainal, M. A., Sahlan, S., \& Ali, M. S. 2015. Micromachined shape-memoryalloy microactuators and their application in biomedical devices. Micromachines, pp. 879-901.

Melzer A., Michitsch St., Konak S., Schaefers G., \& Bertsch Th., 2009. Nitinol in magnetic resonance imaging. Minimally Invasive Therapy \& Allied Technologies. 13(4), pp. 261-271.

Taylor R. H., Menciassi A., Fichtinger G., Fiorini P., Dario P., 2016. Chapter 63 - Medical Robotics and Computer-Integrated Surgery. In: Siciliano B., Khatib O. (eds) Springer Handbook of Robotics (pp. 1657-1684). Cham: Springer.

Haga, Y., Mizushima, M., Matsunaga, T., Esashi, M. 2005. Medical and welfare applications of shape memory alloy microcoil actuators. Smart Mater Struct, 14 , pp. 266-272.

Ho, M. M. 2011. Towards a meso-scale SMA-actuated MRI-compatible neurosurgical robot. IEEE Trans Robot, 2011(99), pp. 1-10.

Augustin, F., Bodner, J.C., Fish, J.H., Muehlmann, G., Schmid, T.A., Wykypiel, H., Wetscher, G.J. 2005. The da Vinci robotic system for general surgical application: A critical interim appraisal. Swiss Med Wkly, 135 45-46, pp. 674-678.

Yarmolenko, P. S., Moon, E. J., Landon, C., Manzoor, A., Hochman, D. W., Viglianti, B. L., \& Dewhirst, M. W. 2011. Thresholds for thermal damage to normal tissues: An update. Int J Hyperther, 27(4), pp. 320-343.

Sars, V. De, Haliyo, S., \& Szewczyk, J. 2010. A practical approach to the design and control of active endoscope. Mechatronics, 20(2), pp. 251-264.

Zaiter, A., Hikmat, O. F., Nafea, M., \& Ali, M. S. M. 2016. Design and fabrication of a novel XY $\theta \mathrm{z}$ monolithic micro-positioning stage driven by NiTi shape-memory-alloy actuators. Smart Mater Struct, 25(10), Article no. 105004 .

Goldman, R. E., Bajo, A., MacLachlan, L. S., Pickens, R., Herrell, S. D., \& Simaan, N. 2013. Design and performance evaluation of minimally invasive telerobotic platform for transurethral surveillence and intervention. IEEE T Bio-med Eng ,60(4), pp. 918-925.

Humbeck, J., Cederstrom, J. 1995. Relationship Between Shape Memory material properties and Applications. J Phys IV, 5, pp. 335-341.

Lobo, P. S., Almeida, J., Guerreiro, L. 2015. Shape Memory Alloy Behaviour: A Review. Procedia Engineer, 114, pp. 776-783. 